OPEN ACCESS

Edited by:

Jiyan Zhang,

Independent Researcher, Beijing,

China

Reviewed by:

Martina Schmidt,

University of Groningen, Netherlands

Zhong Wang,

Soochow University, China

${ }^{*}$ Correspondence:

Kailiang Zhou

zhoukailiang@wmu.edu.cn

Wenfei $\mathrm{N}$

niwenfei@126.com

tThese authors have contributed equally to this work and share first authorship

Specialty section

This article was submitted to Cell Death and Survival,

a section of the journal

Frontiers in Cell and Developmental

Biology

Received: 06 May 2021

Accepted: 12 July 2021

Published: 04 August 2021

Citation:

Hu X, XU Y, Xu H, Jin C, Zhang H, Su H, Li Y, Zhou K and Ni W (2021)

Progress in Understanding

Ferroptosis and Its Targeting for Therapeutic Benefits in Traumatic

Brain and Spinal Cord Injuries.

Front. Cell Dev. Biol. 9:705786.

doi: 10.3389/fcell.2021.705786

\section{Progress in Understanding Ferroptosis and Its Targeting for Therapeutic Benefits in Traumatic Brain and Spinal Cord Injuries}

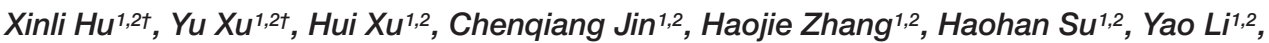 \\ Kailiang $\mathrm{Zhou}^{1,2 *}$ and Wenfei $\mathrm{Ni}^{1,2 *}$
}

${ }^{1}$ Department of Orthopaedics, The Second Affiliated Hospital and Yuying Children's Hospital of Wenzhou Medical University, Wenzhou, China, ${ }^{2}$ Zhejiang Provincial Key Laboratory of Orthopaedics, Wenzhou, China

Acute central nervous system (CNS) trauma, including spinal cord injury (SCl) and traumatic brain injury (TBI), always leads to severe sensory, motor and autonomic nervous system dysfunction due to a series of processes, including cell death, oxidative stress, inflammation, and excitotoxicity. In recent years, ferroptosis was reported to be a type of programmed cell death characterized by the consumption of polyunsaturated fatty acids and the accumulation of membrane lipid peroxides. The processes that induce ferroptosis include iron overload, imbalanced glutathione metabolism and lipid peroxidation. Several studies have indicated a novel association of ferroptosis and acute CNS trauma. The present paper reviews recent studies of the occurrence of ferroptosis, stressing the definition and process of ferroptosis and metabolic pathways related to ferroptosis. Furthermore, a summary of the existing knowledge of the role of ferroptosis in CNS trauma is presented. The aim here is to effectively understand the mechanisms underlying the occurrence of ferroptosis, as well as the relevant effect on the pathophysiological process of CNS trauma, to present a novel perspective and frame of reference for subsequent investigations.

Keywords: ferroptosis, programmed cell death, CNS trauma, spinal cord injury, traumatic brain injury

\begin{abstract}
Abbreviations: CNS, Central nervous system; SCI, Spinal cord injury; TBI, Traumatic brain injury; L-ROS, Reactive oxygen species; L-OOH, Lipid hydroperoxides; L-OH, lipid alcohols; GSH, Glutathione; CoA, Coenzyme A; OH, Soluble hydroxyl; RO, Lipid alkoxy; RSL3, (1S,3R)-RSL3; Hb, Hemoglobin; TFR1, Transferrin receptor 1; DMT1, Divalent metal transporter 1; LIP, Labile iron pool; PUFA, Polyunsaturated fatty acid; ACSL4, Acyl-CoA synthetase long-chain family member 4; LPCAT3, Lysophosphatidylcholine acyl transferase 3; PLs, Phospholipids; BSO, Buthionine sulfoximine; GPX4, Glutathione peroxidase 4; DPI2, Diverse pharmacological inhibitor-2; NAPQI, N-acetyl-p-benzoquinone imine; ROOH, Reduce organic peroxide; $\mathrm{H}_{2} \mathrm{O}_{2}$, Free hydrogen peroxide; L- O-, Alkoxy radicals; Tf, Transferrin; TFR1, Transferrin receptor 1; STEAP3, Sixtransmembrane protein prostate 3; DMT1, Divalent metal transporter 1; PCBP1, Poly(rC)-binding protein; AA, Catalyses arachidonic acid; AdA, Adrenic acid; PE, Phosphatidylethanolamines; LOXs, Lipoxygenase; 15-LOX, 15-lipoxygenase; TEM, Transmission electron microscopy; IREB2, Iron-responsive element-binding protein 2; ACSF2, Acyl-CoA synthetase family member 2; DFO, Deferoxamine; PAC, Proanthocyanidin; ALOX15B, Arachidonate 15-Lipoxygenase Type B; TBARS, Thiobarbituric acid reactive substances; Nrf2, Nuclear factor-erythroid 2-related factor 2; HO-1, Heme oxygenase-1; GSSG, Oxidized glutathione; Lipro-1, Liproxstatin-1; IREB2, Iron Responsive Element Binding Protein 2; PTGS2, Prostaglandinendoperoxide synthase 2; RPL8, Ribosomal Protein L8; CCI, Cortical impact injury; MDA, Malondialdehyde; AA/AdAPE, Arachidonic/adrenic acid-containing-phosphatidylethanolamine; COX-2, Cyclooxygenase-2; IRI, Ischemia-reperfusion injury; MLKL, Mixed lineage kinase domain-like pseudokinase.
\end{abstract}




\section{INTRODUCTION}

Acute central nervous system (CNS) trauma, including spinal cord injury (SCI) and traumatic brain injury (TBI), is a primary cause of mortality and morbidity worldwide ( $\mathrm{Hu}$ et al., 2020). Each year, approximately 50 million individuals worldwide suffer from TBI (Maas et al., 2017). SCI, another form of neurological trauma, has an incidence of over 2.5 million people globally (Le Corre et al., 2018). Regarding the mechanisms associated with acute CNS trauma, existing studies have reported the death of neuronal cells as a vital pathological event in CNS trauma (Shi et al., 2021). The pathophysiology of CNS trauma includes primary injury and secondary injury (Ng and Lee, 2019). Secondary damage mainly includes the release of intracellular and intra-axonal contents, such as glutamate, reactive oxygen species (ROS), and $\mathrm{Fe}^{2+}$ (Hu et al., 2020). Glutamate-induced excitotoxicity is one of the major events in secondary CNS trauma. Excitotoxicity leads to mitochondrial damage and dysfunction, increased ROS release and DNA damage, all of which lead to different types of cell death (Hiebert et al., 2015). Therefore, the ability to control the various cascades of injury-induced cell death during secondary injury is a vital problem that must be solved to develop effective therapies that promote recovery after CNS trauma. Rapidly accumulating evidence indicates that neurons are vulnerable to ferroptotic cell death in response to CNS trauma (Geng et al., 2021).

Compared with other forms of programmed cell death, such as autophagy, pyroptosis and necroptosis, ferroptosis exhibits special morphological and biological features. The morphological characteristics of ferroptosis include the rupture of cell membranes, but chromatin condensation is lacking in the cell nucleus (Čepelak et al., 2020). Using electron microscopy, ferroptosis has been shown to trigger cell death by inducing a loss of mitochondrial cristae, increased density of mitochondrial membrane, and shrunken mitochondria (Neitemeier et al., 2017). The biological characteristics of ferroptosis include the accumulation of ROS and iron. This characteristic is not consistent with autophagy and apoptosis. Although the mechanisms of ferroptosis have been well studied, the possible molecular and pathogenic mechanisms involved in CNS trauma remain unclear, indicating that the elucidation of the mechanisms of ferroptosis is important. This review aims to summarize recently conducted studies on CNS trauma and ferroptosis to determine how ferroptosis modulates CNS trauma.

\section{DEFINITION AND PROCESS OF FERROPTOSIS}

The term ferroptosis emerged in 2012 to describe the form of cell death triggered by the small molecule erastin, thereby inhibiting cystine import, triggering the inactivation of phospholipid peroxidase glutathione peroxidase 4 (GPX4) and consumption of glutathione (Dixon et al., 2012). GPX4 is capable of converting lipid hydroperoxides $(\mathrm{L}-\mathrm{OOH})$ with toxic potential into non-toxic lipid alcohols (L-OH) (Forcina and Dixon, 2019). The inactivation of GPX4 mediated by erastin-induced glutathione (GSH) consumption or treatment with the direct GPX4 inhibitor (1S,3R)-RSL3 (RSL3) leads to preponderant lipid peroxidation, which causes cell death. Ferroptosis was suggested to be involved in pathological processes after CNS trauma (Xie et al., 2019). First, the acute phase of CNS trauma is characterized by immediate hemorrhage (Fletcher-Sandersjöö et al., 2021). In addition, hemoglobin ( $\mathrm{Hb})$, the most abundant protein in blood, is metabolized into ferrous/ferric iron, which causes iron overload at the injury site (Wan et al., 2019). $\mathrm{Fe}^{3+}$ is introduced in the cell through the membrane protein transferrin receptor 1 (TFR1) and subsequently localizes to endosomes (Forcina and Dixon, 2019). Iron reductase is specifically located in endosomes and reduces $\mathrm{Fe}^{3+}$ into $\mathrm{Fe}^{2+}$ (Stockwell et al., 2017). Last, divalent metal transporter 1 (DMT1) transports $\mathrm{Fe}^{2+}$ to the labile iron pool (LIP) in unstable iron pools within the cytoplasm, and large amount of iron is stored within ferritin, a protein complex responsible for iron storage (Harrison and Arosio, 1996). However, under trauma conditions, the LIP, the chemically reactive and kinetically labile pool, releases $\mathrm{Fe}^{2+}$ into the cytoplasm (Ke and Qian, 2007). Excess iron is capable of synthesizing hydroxyl radicals with high oxidizing activities (such as ROS) through the Fenton reaction, in which $\mathrm{Fe}^{2+}$ reacts with hydrogen (Forcina and Dixon, 2019). This process leads to ferroptosis. At the same time, oxidation of polyunsaturated fatty acid (PUFA)containing phospholipids (PLs) the plasma membrane was recently identified as a key process for ferroptosis (Stockwell et al., 2017). Acyl-CoA synthetase long-chain family member 4 (ACSL4) preferentially activates PUFA free fatty acids to generate PUFA-CoAs (Stockwell et al., 2017). Lysophosphatidylcholine acyl transferase 3 (LPCAT3) insert acyl-CoA molecules into lysophosphatidylcholine to synthesize phosphatidylcholine (Doll and Conrad, 2017). Generally, these 2 enzymes are conducive to defining a lipid metabolic pathway critical for inserting PUFAs into membrane PLs and improving susceptibility to ferroptosis (Doll et al., 2017). In addition, ferroptosis is regulated by the GPX4-GSH-cysteine axis. In this axis, vital processes comprise cystine uptake through system $\mathrm{Xc}^{-}$, reducing the processing of phospholipid hydroperoxides into lipid alcohols by GPX4, GSH biosynthesis, and reducing the transformation of cystine into cysteine (Chen J. et al., 2020). This procedure can relieve ferroptosis.

\section{METABOLIC PATHWAYS RELATED TO FERROPTOSIS}

Three metabolic pathways have been shown to be closely associated with the regulation of ferroptosis, including glutathione metabolism, lipid metabolism and iron metabolism (Shen et al., 2020). Thus, we will discuss the relationship between the three metabolic pathways and ferroptosis below. A summary of the metabolic pathways related to ferroptosis is shown in Figure 1. 


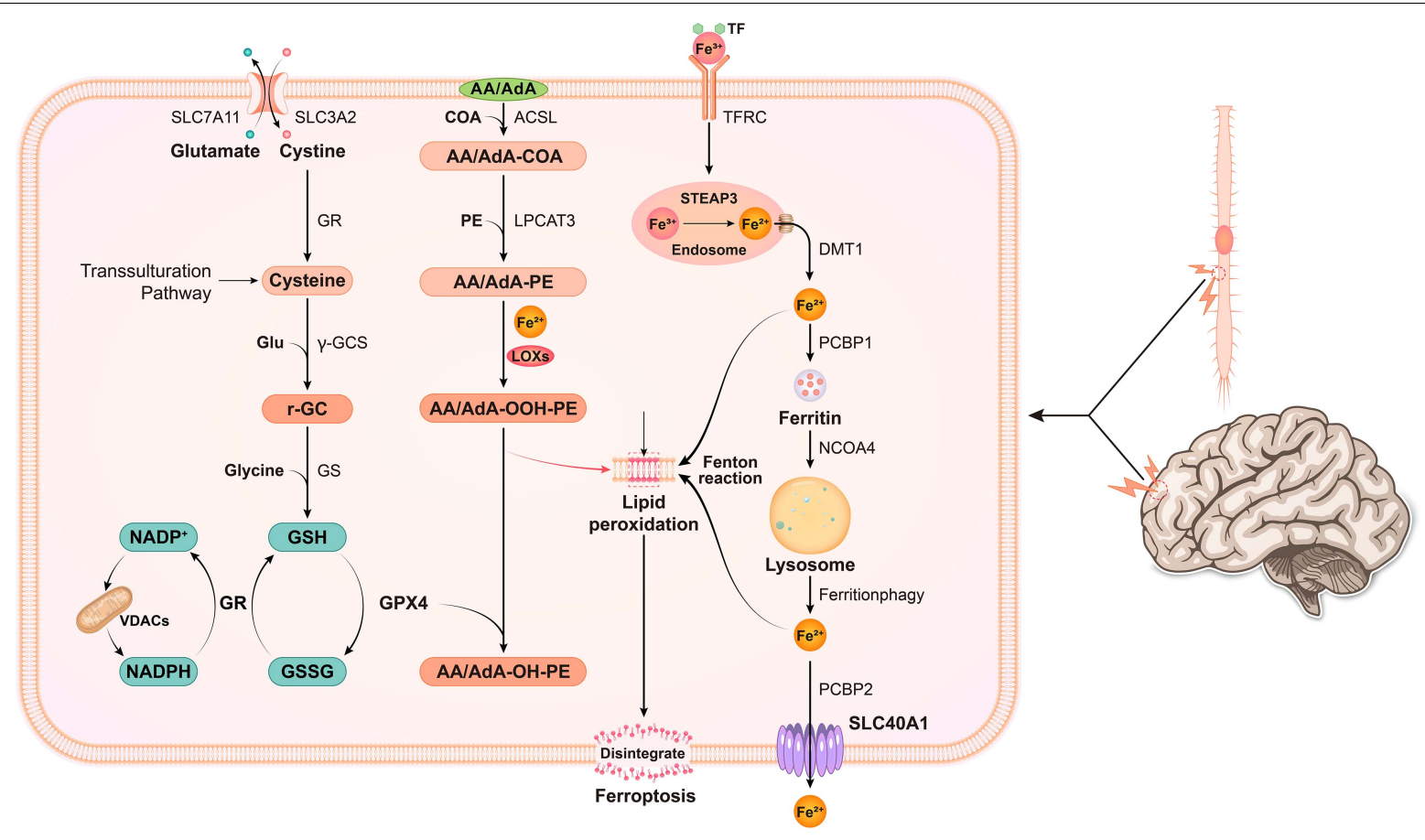

FIGURE 1 | In nerve cells, various pathogenic factors cause abnormal increases in $\mathrm{Fe}^{3+}$ levels, and $\mathrm{Fe}^{3+}$ is then converted into Fe $\mathrm{F}^{2+}$ by STEAP3. Free iron (Fe $\mathrm{ST}^{2+}$ ) is transported from the endosome by DMT1. Some of the free iron is stored in ferritin through PCBP1, and some is transported out of the cell through SLC40A1. Free iron is released upon ferritin degradation via the ferritinophagy pathway, which is mediated by NCOA4, and might cause lipid ROS. System xc- is a cystine/glutamate antiporter. Intracellular cystine is reduced to cysteine for the biosynthesis of GSH. When cystine is inside the cell, cystine is reduced to cysteine, and the level of cysteine is supplemented by the transsulfuration pathway. Subsequently, cysteine is used for the biosynthesis of GSH. GPX4 uses two GSH molecules as electron donors to reduce phospholipid hydroperoxides (PL-OOH) to the corresponding alcohols, leaving GSSG (oxidized GSH) as a by-product. Then, GSSG is recycled by GSH reductase in an NADPH-dependent manner. The ion selectivity of VDAC determines whether cations or anions enter the mitochondria, thus affecting the oxidation state of NADH. ACSL4 is required to activate PUFAs, especially AA and AdA, to AA-CoA, and AdA acyl-CoA derivatives. These derivatives are esterified by LPCA T3 into AA-PE and AdA-PE, which are then catalyzed by the iron-containing enzyme lipoxygenase (LOX) to generate fatty acid hydroperoxides, thus leading to ferroptosis.

\section{Glutathione Metabolism Is Related to Ferroptosis}

System $\mathrm{Xc}^{-}$is a $\mathrm{Na}^{+}$-dependent cysteine-glutamate antiporter located in the membrane that is composed of SLC3A2 and SLC7A11 (xCT) and responsible for the exchange of intracellular glutamate for extracellular cystine at a 1:1 ratio (Shen et al., 2020). If transported in the cell, cystine is reduced to cysteine via the GSH synthesis process (Cepelak et al., 2020). Among the 3 amino acids identified in GSH, cysteine is present at the lowest abundance, and thus it is recognized as the ratelimiting residue to achieve de novo GSH synthesis (Dixon et al., 2014). Cysteine is synthesized from methionine in several cell types through transsulfuration. The cysteine-glutathione channel was suggested to be a crucial upstream signaling pathway regulating ferroptosis (Dixon et al., 2014). System $\mathrm{Xc}^{-}$, which is not regulated by ATP, is activated by high concentrations of intracellular glutamate. Thus, it is particularly sensitive to the extracellular glutamate concentration. Millimolar concentrations of extracellular glutamate block system $\mathrm{Xc}^{-}$activity and trigger oxidation-related cell death, which was previously considered oxytosis (Tobaben et al., 2011). Increased extracellular glutamate levels occurring following numerous types of brain trauma suppress system $\mathrm{Xc}^{-}$transport, thereby inducing ferroptosis (Latunde-Dada, 2017). The direct inhibition of system $\mathrm{Xc}^{-}$ was recently reported to induce cell death by decreasing cysteine uptake, thereby causing GSH depletion and impairing the ability of the cell to resist oxidation-related stress (Gout et al., 2001). Moreover, several studies have shown that GSH depletion induced by buthionine sulfoximine (BSO), diverse pharmacological inhibitor-2 (DPI2), an active metabolite of acetaminophen, and N-acetyl-p-benzoquinone imine (NAPQI) is associated with ferroptosis. Glutathione peroxidase 4 (GPX4) is a member of the GPX family that is involved in ferroptosis. GPX4, together with GSH, reduce organic peroxide ( $\mathrm{ROOH}$ ) or free hydrogen peroxide $\left(\mathrm{H}_{2} \mathrm{O}_{2}\right)$ to the corresponding alcohols and water. Additionally, the catalytic cycle of GPX4 consumes $2 \mathrm{GSH}$ molecules to generate oxidized glutathione (GSSG) (Wu et al., 2019). GSSG can be recycled as GSH through GSH reductase in an NADPH-dependent manner (Farina and Aschner, 2019). L-OOH formation and $\mathrm{L}-\mathrm{OOH}$ reduction into $\mathrm{L}-\mathrm{OH}$ are balanced constantly by GPX4 (Li et al., 2021). L-OOH perform oxidation using $\mathrm{Fe}^{2+}$ to yield significantly reactive alkoxy radicals (L-O-) (Shen et al., 2020). The mentioned radicals directly damage nearby PUFAs via a 
free radical-mediated chain reaction process (Xie et al., 2016). Following the loss or inactivation of GPX4, L-OOH accumulates in larger concentrations than normal, thereby causing more significant L-O- production and possibly resulting in catastrophic membrane injury (Wu et al., 2019).

\section{Iron Metabolism Is Related to Ferroptosis}

Iron is a vital nutrient to maintain physiological function. Iron has two states in humans, free and bound iron. The majority of bound iron is in the form of ferritin, for example, iron-sulfur nanoclusters and hemoglobin, whereas free iron, or non-bound iron, is mainly present inside non-iron-sulfur nanoclusters or haem (Chen Y. et al., 2020). Free iron consists of ferric iron and ferrous iron. Although researchers have not clearly determined how iron affects the lipid oxidation process to promote ferroptosis, iron is an essential component of oxidative cell death (Tang et al., 2021). Ferroportin, ferritin, transferrin and other proteins that contain iron for iron degradation, utilization, storage, and uptake help facilitate ferroptosis (Cotticelli et al., 2019). A large amount of free $\mathrm{Fe}^{3+}$ in the blood forms a complex composed of extracellular transferrin (Tf) and transferrin receptor 1 (TFR1) in transplanted cells and the cell membrane during endocytosis (Biasiotto et al., 2019). Next, based on the acidic $\mathrm{pH}$ environment in the endosome, $\mathrm{Fe}^{3+}$ is degraded into the highly reactive $\mathrm{Fe}^{2+}$ by the six-transmembrane protein prostate 3 (STEAP3) (Shen et al., 2020). Through a process mediated by divalent metal transporter 1 (DMT1, also known as SLC11A2), $\mathrm{Fe}^{2+}$ translocates from endosomes into the cytoplasm and subsequently forms an iron pool that is not stable inside the cytoplasm (Ke and Qian, 2007). $\mathrm{Fe}^{2+}$ inside the iron pool undergoes partial delivery to ferritin by directly binding to Poly(rC)-binding protein 1 (PCBP1) to protect cells and tissues from iron-induced injury, whereas some $\mathrm{Fe}^{2+}$ also binds to PCBP2 and then is removed from the cell via SLC40A1 located on the cell membrane (Tang et al., 2021). In a normal environment, the concentration of intracellular iron remains steady. If the balance is disrupted, such as iron under overload, excessive iron and divalent ferrous ion $\mathrm{Fe}^{2+}$ are precisely capable of reacting with $\mathrm{ROOH}$ or $\mathrm{H}_{2} \mathrm{O}_{2}$ to yield soluble lipid alkoxy (RO-) or hydroxyl (HO-) radicals, respectively (Tang et al., 2021). These substances are the source of major ROS generated from $\mathrm{Fe}^{2+}$ inside cells through a process known as the Fenton reaction (Dixon and Stockwell, 2014). They easily acquire electrons from other molecules to facilitate lipid peroxide formation and induce ferroptosis.

\section{Lipid Metabolism Is Associated With Ferroptosis}

The accumulation of lipid peroxidation is suggested to be critical in the execution phase, with PUFAs being vital (Doll and Conrad, 2017). New long-chain fatty acids are converted to $\mathrm{CoA}$ to be incorporated into phospholipids (PLs) (Liang et al., 2019). A vital enzyme regulating ferroptosis, ACSL4, catalyzes arachidonic acid (AA) and adrenic acid (AdA) acylation reactions (Doll et al., 2017). Upon catalysis by LPCTA3, the acetylation product is combined with phosphatidylethanolamines (PE) in the membrane PL to produce PE-AdA and PE-AA (Kagan et al., 2017). After AA/AdA-PE is generated, activated lipoxygenase (LOXs), especially 15-lipoxygenase (15-LOX), catalyze the transformation of AA/AdA-PE into the proferroptotic lipid peroxidation product AA/AdA-OOH-PE, which leads to lipid injury that is impossible to repair and a permeabilized membrane (Doll and Conrad, 2017). In addition, some studies suggest that the function of the LOXs family of enzymes depends on iron (Magtanong and Dixon, 2018; Wu et al., 2019). However, at present, no conclusive evidence for the iron dependence of LOX is available, which deserves further study. The method of eliminating AA/AdA-OOH-PE only depends on GPX4, and this enzyme exhibits the ability to reduce toxic, membranous lipid hydroperoxides into non-toxic lipid alcohols (Yang et al., 2014). Thus, the GPX4 inactivation process promotes the accumulation of lipid peroxidation and ferroptosis initiation.

\section{THE EFFECT OF FERROPTOSIS ON TBI AND SCI}

When external forces are applied to the brain and spinal cord, blood vessels rupture, the blood-brain barrier and blood-spinal cord barrier are destroyed, and blood leakage occurs inside the brain and spinal cord (Wan et al., 2019). Accordingly, iron from hemoglobin present in the blood is released and induces iron overload (Shen et al., 2020). Divalent ferrous ion $\mathrm{Fe}^{2+}$ produces excess ROS through the Fenton reaction. The major effect exerted by ROS is oxidative injury to biofilms containing PUFAs (Xie et al., 2016). Meanwhile, cholesterol and PUFAs are abundant in neuronal membranes and exhibit high susceptibility to oxidizing processes induced by ROS (Shichiri, 2014). Moreover, the capability of neurons to scavenge ROS through an autonomous approach is limited (Hiebert et al., 2015). Thus, neurons exhibit exceptional vulnerability to iron overload. TBI and SCI share many of the same pathophysiological features. The present review investigates the mechanisms by which iron and ferroptosis affect TBI and SCI to determine the effects of iron metabolic processes on TBI and SCI and to identify a novel and feasible treatment for SCI and TBI targeting ferroptosis and iron (Table 1).

\section{The Effect of Ferroptosis on SCI}

After spinal cord injury, the spinal cord exhibits substantial hemorrhaging, erythrocyte aggregation, cell rupture, and hemolysis and local iron overload (Derry et al., 2020). Moreover, stress induces the production of a large amount of ROS and increases the excitotoxicity of glutamate (Kang et al., 2020). All of these factors induce ferroptosis. Ferroptosis signaling critically affect SCI. Mitochondrial morphology is a vital criterion that supports a role for ferroptosis in SCI. Using transmission electron microscopy (TEM), Yao et al. revealed variations within the mitochondria of ferroptotic cells (Yao et al., 2019). They also observed noticeable changes in the levels of ferroptosis markers such as GPX4, $\mathrm{xCT}$, and glutathione in spinal cord tissues from rats with SCI. In addition, using real-time 
TABLE 1 | Ferroptosis-associated drugs used to treat TBI and SCI.

\begin{tabular}{|c|c|c|c|}
\hline Disease & Drug & Target & Potential mechanism \\
\hline \multirow[t]{4}{*}{$\mathrm{SCl}$} & Deferoxamine & Iron chelator & $\begin{array}{l}\text { Increased xCT, GSH, and GPX } 4 \\
\text { levels }\end{array}$ \\
\hline & SRS 16-86 & $\begin{array}{l}\text { Small-molecule, } \\
\text { ferroptosis-specific } \\
\text { inhibitor }\end{array}$ & $\begin{array}{l}\text { Upregulated GPX } 4, \mathrm{GSH} \text {, and } \\
\text { xCT levels }\end{array}$ \\
\hline & Proanthocyanidin & $\begin{array}{l}\text { Lipid peroxidation } \\
\text { inhibitor }\end{array}$ & $\begin{array}{l}\text { Upregulated GPX } 4 \text {, GSH, } \\
\mathrm{HO}-1 \text {, and xCT levels }\end{array}$ \\
\hline & Liproxstatin-1 & $\begin{array}{l}\text { Lipid peroxidation } \\
\text { inhibitor }\end{array}$ & $\begin{array}{l}\text { Suppresses mitochondrial lipid } \\
\text { peroxidation, reduces MDA } \\
\text { levels, and upregulates GSH, } \\
\text { GPX4, and FSP1 }\end{array}$ \\
\hline \multirow[t]{3}{*}{ TBI } & Baicalein & $\begin{array}{l}\text { 12/15-lipoxygenase } \\
\text { inhibitor }\end{array}$ & $\begin{array}{l}\text { Suppressed the } \\
\text { 15-lipoxygenase pathway }\end{array}$ \\
\hline & miR-212-5p agomir & PTGS2 inhibition & $\begin{array}{l}\text { Suppressed the enzyme } \\
\text { catalyzing lipid oxidation }\end{array}$ \\
\hline & Ferrostatin-1 & $\begin{array}{l}\text { Lipid peroxidation } \\
\text { inhibitor }\end{array}$ & Reduces iron deposition \\
\hline
\end{tabular}

polymerase chain reaction, they detected increased mRNA levels of ferroptosis-related genes, such as iron-responsive element-binding protein 2 (IREB2) and acyl-CoA synthetase family member 2 (ACSF2). As suggested by the results described here, ferroptosis critically affects SCI. Reactive astrocytes, which are the major component of the glial scar and function as a chemical and physical barrier to axon regeneration, are associated with the failure of axon regeneration in individuals with SCI (Patil et al., 2021). Deferoxamine (DFO), an iron chelator, is capable of suppressing ferroptosis triggered by the increase in the activity of the $\mathrm{Xc}^{-} / \mathrm{GPX} 4$ axis in SCI, and the antigliotic effect of DFO was consistent with its effect on GPX4 upregulation (Derry et al., 2020). This result indicated a potential link between gliosis and ferroptosis following SCI, which requires further research. Subsequently, ferroptosis has been extensively studied in spinal cord injury. Zhang et al. (2019) investigated the noticeable effect of the ferroptosis inhibitor SRS 16-86 on attenuating ferroptosis in an animal model of SCI. According to the authors of this study, more common mitochondrial morphological characteristics were observed upon SRS 16-86 treatment at $24 \mathrm{~h}$, indicating a greater volume and larger mitochondrial cristae in contrast to the SCI-vehicle cohort. Regarding the mechanisms by which SRS 16-86 regulates ferroptosis, this study has also illustrated that SRS 16-86 suppresses ferroptosis by upregulating xCT, GPX4, and GSH and suppressing lipid peroxidation. Furthermore, the authors found that SRS 16-86 treatment reduced astrogliosis and increased neuronal survival after SCI. Nuclear factor-erythroid 2-related factor 2 (Nrf2), a master regulator of the cellular antioxidant response, involves in regulating dozens of genes which related to ferroptosis, such as GPX4 and xCT (Abdalkader et al., 2018). Additionally, oxygenase-1 (HO-1) plays a critical role in heme metabolism which have protective or detrimental effects during ferroptosis (Chiang et al., 2018). In addition, thiobarbituric acid reactive substances (TBARS) is commonly quantified to assess the extent of lipid peroxidation (Zhou et al., 2020). Zhou et al. (2020) investigated another ferroptosis inhibitor, proanthocyanidin (PAC), which attenuates ferroptosis through different mechanisms after SCI. According to their results, PAC treatment noticeably reduced the levels of iron, ALOX15B, ACSL4, and TBARS and increased heme HO-1, Nrf2, GPX4, and GSH levels in SCI models. These results reveal the protective effects of the PAC treatment on ferroptosis through iron chelation and antioxidant activity. LPCAT3 and ACSL4 contribute to cellular lipid generation and essentially modulate ferroptosis (Yuan et al., 2016). PAC treatment insignificantly altered the increase in LPCAT3 levels inside the injured spinal cord, but it decreased ACSL4 levels in the spinal cord (Kang et al., 2020). Thus, researchers have not clearly determined whether PACs decrease arachidonic acid or adrenic acid levels to inhibit ferroptosis. Hence, this topic requires in-depth investigation. Alox15B is involved in the cellular lipid synthesis process and is needed for ferroptosis. Alox15B was reported to be widely expressed in neutrophils and macrophages (Archambault et al., 2018; Snodgrass and Brüne, 2019). The decrease in Alox15B levels induced by the PAC treatment after SCI suggests that Alox15B is likely a cellular target of PACs since it is extensively expressed in neutrophils and macrophages. Fan et al. (2021) reported that liproxstatin-1 (Lipro-1) is an inhibitor that effectively blocks ferroptosis and elucidated the possible pharmacological effects of Lipro-1 on suppressing ferroptosis and its signaling pathways. First, GPX4 is a critical factor regulating ferroptosis (Yang et al., 2014). In the spinal cord, GPX4 is primarily expressed in neurons and oligodendrocytes, but not in astrocytes (Hu et al., 2019). Interestingly, in contrast to a neuronal cytoplasmic localization, GPX4 localizes to the nucleus in oligodendrocytes in vivo ( $\mathrm{Hu}$ et al., 2019). GPX4 consumes GSH to inhibit lipid peroxidation, particularly lipid hydroperoxidation, in biological membranes (Brigelius-Flohé and Maiorino, 2013). On the one hand, Lipro-1 restored GPX4 to normal levels in ferroptotic oligodendrocytes to inhibit the ferroptosis signal within the nucleus (Imai et al., 2017). On the other hand, Lipro-1 increased GSH levels to enhance the anti-ferroptosis system (Fan et al., 2021). In addition, one existing study reported that Lipro-1 was capable of 
decreasing mitochondrial ROS levels (Feng et al., 2019). Based on these results, Lipro-1 may inhibit oligodendrocyte ferroptosis. After SCI, damage to oligodendrocytes induced by ferroptosis potentially aggravates demyelination. However, Lipro-1 inhibits oligodendrocyte ferroptosis to repair SCI. In summary, Lipro-1 may be a potential drug for the treatment of SCI.

\section{The Effect of Ferroptosis on TBI}

Spinal cord injury and TBI have similar pathophysiological mechanisms, and ferroptosis occurs in TBI. Xie et al. (2019) revealed that the $\mathrm{mRNA}$ levels of genes related to ferroptosis [such as Ribosomal Protein L8(RPL8), Iron Responsive Element Binding Protein 2(IREB2), Prostaglandin-endoperoxide synthase 2 (PTGS2), and ATP5G3] were remarkably increased within the cortex of a controlled cortical impact injury (CCI) mouse model compared to sham animal model groups. Additionally, GPX activity decreased noticeably after $6 \mathrm{~h}, 1$ and 3 days. The malondialdehyde (MDA) concentration and lipid ROS levels increased noticeably at $6 \mathrm{~h}$, peaked on day 3 and returned to baseline on day 7. Mitochondrial morphological characteristics are some of vital factors that support ferroptosis. Meanwhile, using TEM, the authors observed shrunken mitochondria in the neuronal soma 3 days after TBI, thus providing powerful evidence for the appearance of ferroptosis after TBI. As proven in subsequent studies, ferroptosis mainly causes severe secondary injury after TBI, and Ferrostatin-1 reduces iron accumulation, attenuates neuronal degeneration in mouse with TBI and ameliorates TBI-induced motor and cognitive deficits (Wu et al., 2019). As confirmed by the aforementioned results, ferroptosis plays an important role in TBI (Xie et al., 2019). Kenny et al. (2019) added RSL3 (an inhibitor of GPX4) to cultures of HT22 cells and observed increased cell death. The author and colleagues discovered that baicalein effectively attenuated the oxidation of arachidonic/adrenic acidcontaining-phosphatidylethanolamine (AA/AdA-PE), a key step in ferroptosis, in a rat CCI model. Moreover, miR-212-5p is likely to prevent ferroptotic neuronal death in a mouse CCI model partially by targeting PTGS2 (Xiao et al., 2019). Xiao et al. (2019) investigated whether miR-212-5p alters ferroptotic neuronal death in mice with TBI and detected ferrous ion and malondialdehyde accumulation (MDA), the expression levels of the ferroptosis-related molecules at $6,12,24,48$, and $72 \mathrm{~h}$ and significant upregulation of GPX4 and ACSL4 expression was observed at $6 \mathrm{~h}$ following in a mouse CCI model. In this study, the authors reported a significant association between ferroptosis and miR-212-5p expression in a mouse CCI model. The possible effect of miR-212-5p on the ferroptosis process was illustrated. The authors assessed the ferroptosis-inducing activity of RSL3 in HT-22 and Neuro-2a cell lines. Then, miR212-5p overexpression was induced by transfecting HT-22 and Neuro-2a cells with a miR-212-5p mimic and its expression was silenced by transfecting a miR-212-5p inhibitor. Comparison with the cells transfected with the mimic negative control, miR-212-5p overexpression reduced the cell death rate (Xiao et al., 2019). According to the results, miR-212-5p critically controls ferroptosis. Using a dual-luciferase assay, the authors found that PTGS2 may be a target gene with possible miR212-5p binding sites. PTGS2, also known as cyclooxygenase-2
(COX-2), is a vital enzyme in prostaglandin biosynthesis that simultaneously produces dioxygenase and peroxidase (Amadio et al., 2017). Cyclooxygenases catalyze lipid oxidation, which is involved in ferroptosis. The current studies on miRNA-mediated regulation of ferroptosis still have some deficiencies. First, the cell type-specific expression of miR-212-5p has not been clearly determined. In-depth research should be carried out to determine its expression pattern. Second, further research is needed to determine whether other miRNAs regulate ferroptosis in TBI. To date, few studies have been conducted on this topic. Thus, it is a very promising direction.

\section{DISCUSSION AND PERSPECTIVES: WILL FERROPTOSIS BE A FUTURE DIRECTION?}

Here, we focus on the effects and therapeutic potential of ferroptosis in a wide range of acute CNS trauma conditions, including TBI and SCI. Although considerable progress has been achieved in the study of ferroptosis, many problems remain to be solved. The pharmacologic effects of molecules inhibit ferroptosis maliy lie at the iron, amino acid and lipid metabolism. Currently, studies on the pharmacologic inhibition of ferroptosis in CNS trauma are limited. Therefore, it's necessary to found other potential drugs to depress ferroptosis in SCI and TBI. In other neurological diseases, many drugs have been proved to alleviate ferroptosis and promote nerve repair, such as edaravone and $\mathrm{N}$-acetylcysteine. It was reported that edaravone inhibited ferroptosis by scavenging chain-initiating water-soluble radicals and chain-carrying lipid peroxyl radicals due to its amphiphilicity, in ischemic stroke and amyotrophic lateral sclerosis (Homma et al., 2019; Spasić et al., 2020). Moreover, it was found that $N$-acetylcysteine reduced neuronal ferroptosis and improved behavior following intracerebral hemorrhage in mice through neutralizing nuclear ALOX5-derived toxic lipid species which is involved in the cellular lipid synthesis process and is needed for ferroptosis (Karuppagounder et al., 2018). However, the effects of above drugs involving ferroptosis regulation have not been investigated in CNS trauma. So whether the drugs can inhibit ferroptosis in TBI and SCI remains to be further studied.

The relationship between ferroptosis and other forms of cell death is unknown. For example, the membrane lipid composition regulates the processes of ferroptosis and necroptosis. In a mouse kidney ischemia-reperfusion injury (IRI) model, Muller et al. observed increased time-dependent sensitivity to necroptosis in ACSL4-knockout cells using CRISPR/Cas9based genome-editing technology (Müller et al., 2017). Mixed lineage kinase domain-like pseudokinase (MLKL) knockout sensitized cells to ferroptotic death. Thus, necroptosis and ferroptosis are intertwined in murine renal IRI. MLKL drives basal resistance to ferroptosis by depleting PUFAs, and ACSL4 drives basal resistance to necroptosis by making the membrane less susceptible to MLKL-driven membrane permeabilization (Chen Y. et al., 2020). In addition, autophagy is associated with ferroptosis. Some scholars found that heat shock protein 90, an evolutionarily conserved and ubiquitously expressed molecular 
chaperone, participated in ferroptosis by regulating the levels of Lamp-2a in the chaperone-mediated autophagy pathway (Thayyullathil et al., 2021). Other scholars also found that autophagy degrades ferritin in neurons to increase intracellular free iron levels and then promote the occurrence of ferroptosis in a rat model of subarachnoid hemorrhage (Patil et al., 2021). Moreover, autophagy participates in the downstream execution of ferroptosis (Thayyullathil et al., 2021). According to Ma et al. (2017) ferroptosis and autophagy do not regulate each other, whereas they were triggered by iron-dependent ROS. Indeed, all of these results were obtained from animal models of other diseases. Further studies are needed to explore whether consistent changes occur in CNS trauma and the association of ferroptosis and other forms of cell death.

Given the complexity of CNS trauma, the biochemical regulation and sensitivity of various cell types, such as oligodendrocytes, microglia, astrocytes, or neurons, to ferroptosis also require discussion. Previous studies have confirmed that cell death after CNS trauma is an important cause of the loss of nerve function (Hu et al., 2020; Shi et al., 2021). Different cell types, such as neurons, astrocytes, microglia, and oligodendrocytes, play various roles in CNS trauma. Studies of the sensitivity of different cell types to ferroptosis are crucial. Recent studies may provide some answers. Scholars have detected lower GSH levels in oligodendrocytes than in astrocytes; thus, the GSH/GPX4 system is insufficient to prevent lipid peroxidation (Thorburne and Juurlink, 1996). Additionally, the total iron content in oligodendrocyte precursor cells is much higher than that in astrocytes. Iron is essential for ferroptosis because of its ability to generate ROS via redox reactions (Jiang et al., 2021). For these two reasons, oligodendrocytes are more sensitive to ferroptosis. In addition, the expression of GPX4 is downregulated in subjects with SCI and TBI. Thus, oligodendrocytes are likely to undergo ferroptosis in individuals with these diseases, which would subsequently aggravate demyelination. Furthermore, Chen L. et al. (2015) found that GPX4 ablation significantly increased the number of reactive astrocytes, which led to astrogliosis. Their results showed an increased number of astrocytes following the induction of ferroptosis, resulting in scar formation. However, this result is

\section{REFERENCES}

Abdalkader, M., Lampinen, R., Kanninen, K. M., Malm, T. M., and Liddell, J. R. (2018). Targeting Nrf2 to suppress ferroptosis and mitochondrial dysfunction in neurodegeneration. Front. Neurosci. 12:466. doi: 10.3389/fnins.2018.00466

Amadio, P., Tarantino, E., Sandrini, L., Tremoli, E., and Barbieri, S. S. (2017). Prostaglandin-endoperoxide synthase-2 deletion affects the natural trafficking of Annexin A2 in monocytes and favours venous thrombosis in mice. Thromb. Haemost. 117, 1486-1497. doi: 10.1160/th16-12-0968

Archambault, A. S., Turcotte, C., Martin, C., Provost, V., Larose, M. C., Laprise, C., et al. (2018). Comparison of eight 15-lipoxygenase (LO) inhibitors on the biosynthesis of 15-LO metabolites by human neutrophils and eosinophils. PLoS One 13:e0202424. doi: 10.1371/journal.pone.0202424

Biasiotto, G., Filosto, M., and Zanella, I. (2019). Editorial: iron and neurodegeneration. Front. Neurosci. 13:1382. doi: 10.3389/fnins.2019.01382

Brigelius-Flohé, R., and Maiorino, M. (2013). Glutathione peroxidases. Biochim Biophys Acta 1830, 3289-3303. doi: 10.1016/j.bbagen.2012.11.020 opposite to the phenomenon by which ferroptosis leads to cell death. Thus, further study is needed to explore the sensitivity of astrocytes to ferroptosis. Many experiments have shown that neurons are very sensitive to ferroptosis. Treatment with the ferroptosis inhibitor ferrostatin-1 significantly reduces neuronal cell death (Xie et al., 2019). The role of microglia in ferroptosis has been less well studied. Therefore, these cells will be the focus of subsequent studies.

\section{CONCLUSION}

Ferroptosis, a novel type of cell death, has recently aroused substantial interest. Studies of ferroptosis have achieved significant progress. The ferroptosis mechanism mainly includes iron toxicity, inactivation of GPX4 and system $\mathrm{Xc}^{-}$, ROS accumulation and ultimately lipid peroxidation. Nevertheless, insights into ferroptosis in CNS trauma remain preliminary, and a number of problems remain to be studied.

\section{AUTHOR CONTRIBUTIONS}

$\mathrm{XH}$ and $\mathrm{YX}$ searched and reviewed literature and drafted the manuscript and revision. HX and CJ discussed and revised the manuscript. HZ, HS, and YL provided the critical comments. $\mathrm{WN}$ and $\mathrm{KZ}$ designed and formulated the review theme and revised and finalized the manuscript. All authors contributed to the article and approved the submitted version.

\section{FUNDING}

This work was supported by grants from the Natural Science Foundation of China (Nos. 82072192 and 81601705 to KZ), Zhejiang Provincial Medicine and Health Technology Project (No. 2017KY472 to KZ and No. 2015RCB022 to HX), Public Welfare Technology Research Project of Zhejiang Province (No. LGF20H150003 to KZ), and Zhejiang Provincial Natural Science Foundation (Nos. LY17H060009 and Y21H060050 to WN).

Čepelak, I., Dodig, S., and Dodig, D. (2020). Ferroptosis: regulated cell death. Arh. Hig. Rada. Toksikol. 71, 99-109. doi: 10.2478/aiht-2020-71-3366

Chen, J., Wang, Y., Wu, J., Yang, J., Li, M., and Chen, Q. (2020). The potential value of targeting ferroptosis in early brain injury after acute CNS disease. Front. Mol. Neurosci. 13:110. doi: 10.3389/fnmol.2020.00110

Chen, L., Hambright, W. S., Na, R., and Ran, Q. (2015). Ablation of the ferroptosis inhibitor glutathione peroxidase 4 in neurons results in rapid motor neuron degeneration and paralysis. J. Biol. Chem. 290, 28097-28106. doi: 10.1074/jbc. M115.680090

Chen, Y., Liu, S., Li, J., Li, Z., Quan, J., Liu, X., et al. (2020). The latest view on the mechanism of ferroptosis and its research progress in spinal cord injury. Oxid. Med. Cell. Longev. 2020:6375938. doi: 10.1155/2020/6375938

Chiang, S. K., Chen, S. E., and Chang, L. C. (2018). A dual role of heme Oxygenase1 in cancer cells. Int. J. Mol. Sci. 20:39. doi: 10.3390/ijms20010039

Cotticelli, M. G., Xia, S., Lin, D., Lee, T., Terrab, L., Wipf, P., et al. (2019). Ferroptosis as a novel therapeutic target for Friedreich's ataxia. J. Pharmacol. Exp. Ther. 369, 47-54. doi: 10.1124/jpet.118.252759 
Derry, P. J., Vo, A. T. T., Gnanansekaran, A., Mitra, J., Liopo, A. V., Hegde, M. L., et al. (2020). The chemical basis of intracerebral hemorrhage and cell toxicity with contributions from eryptosis and ferroptosis. Front. Cell. Neurosci. 14:603043. doi: 10.3389/fncel.2020.603043

Dixon, S. J., and Stockwell, B. R. (2014). The role of iron and reactive oxygen species in cell death. Nat. Chem. Biol. 10, 9-17. doi: 10.1038/nchembio.1416

Dixon, S. J., Lemberg, K. M., Lamprecht, M. R., Skouta, R., Zaitsev, E. M., Gleason, C. E., et al. (2012). Ferroptosis: an iron-dependent form of nonapoptotic cell death. Cell 149, 1060-1072. doi: 10.1016/j.cell.2012.03.042

Dixon, S. J., Patel, D. N., Welsch, M., Skouta, R., Lee, E. D., Hayano, M., et al. (2014). Pharmacological inhibition of cystine-glutamate exchange induces endoplasmic reticulum stress and ferroptosis. Elife 3:e02523. doi: 10.7554/eLife. 02523

Doll, S., and Conrad, M. (2017). Iron and ferroptosis: a still ill-defined liaison. IUBMB Life 69, 423-434. doi: 10.1002/iub.1616

Doll, S., Proneth, B., Tyurina, Y. Y., Panzilius, E., Kobayashi, S., Ingold, I., et al. (2017). ACSL4 dictates ferroptosis sensitivity by shaping cellular lipid composition. Nat. Chem. Biol. 13, 91-98. doi: 10.1038/nchembio.2239

Fan, B. Y., Pang, Y. L., Li, W. X., Zhao, C. X., Zhang, Y., Wang, X., et al. (2021). Liproxstatin-1 is an effective inhibitor of oligodendrocyte ferroptosis induced by inhibition of glutathione peroxidase 4. Neural Regen. Res. 16, 561-566. doi: 10.4103/1673-5374.293157

Farina, M., and Aschner, M. (2019). Glutathione antioxidant system and methylmercury-induced neurotoxicity: an intriguing interplay. Biochim. Biophys. Acta Gen. Subj. 1863:129285. doi: 10.1016/j.bbagen.2019.01.007

Feng, Y., Madungwe, N. B., Imam Aliagan, A. D., Tombo, N., and Bopassa, J. C. (2019). Liproxstatin-1 protects the mouse myocardium against ischemia/reperfusion injury by decreasing VDAC1 levels and restoring GPX4 levels. Biochem. Biophys. Res. Commun. 520, 606-611. doi: 10.1016/j.bbrc.2019. 10.006

Fletcher-Sandersjöö, A., Thelin, E. P., Maegele, M., Svensson, M., and Bellander, B. M. (2021). Time course of hemostatic disruptions after traumatic brain injury: a systematic review of the literature. Neurocrit. Care 34, 635-656. doi: 10.1007/s12028-020-01037-8

Forcina, G. C., and Dixon, S. J. (2019). GPX4 at the crossroads of lipid homeostasis and ferroptosis. Proteomics 19:e1800311. doi: 10.1002/pmic.201800311

Geng, Z., Guo, Z., Guo, R., Ye, R., Zhu, W., and Yan, B. (2021). Ferroptosis and traumatic brain injury. Brain Res. Bull. 172, 212-219. doi: 10.1016/j. brainresbull.2021.04.023

Gout, P. W., Buckley, A. R., Simms, C. R., and Bruchovsky, N. (2001). Sulfasalazine, a potent suppressor of lymphoma growth by inhibition of the $\mathrm{x}(\mathrm{c})$ - cystine transporter: a new action for an old drug. Leukemia 15, 1633-1640. doi: 10.1038/sj.leu.2402238

Harrison, P. M., and Arosio, P. (1996). The ferritins: molecular properties, iron storage function and cellular regulation. Biochim. Biophys. Acta 1275, 161-203. doi: 10.1016/0005-2728(96)00022-9

Hiebert, J. B., Shen, Q., Thimmesch, A. R., and Pierce, J. D. (2015). Traumatic brain injury and mitochondrial dysfunction. Am. J. Med. Sci. 350, 132-138. doi: 10.1097/maj.0000000000000506

Homma, T., Kobayashi, S., Sato, H., and Fujii, J. (2019). Edaravone, a free radical scavenger, protects against ferroptotic cell death in vitro. Exp. Cell Res. 384:111592. doi: 10.1016/j.yexcr.2019.111592

Hu, C. L., Nydes, M., Shanley, K. L., Morales Pantoja, I. E., Howard, T. A., and Bizzozero, O. A. (2019). Reduced expression of the ferroptosis inhibitor glutathione peroxidase- 4 in multiple sclerosis and experimental autoimmune encephalomyelitis. J. Neurochem. 148, 426-439. doi: 10.1111/jnc.14604

Hu, X., Chen, H., Xu, H., Wu, Y., Wu, C., Jia, C., et al. (2020). Role of pyroptosis in traumatic brain and spinal cord injuries. Int. J. Biol. Sci. 16, 2042-2050. doi: $10.7150 /$ ijbs. 45467

Imai, H., Matsuoka, M., Kumagai, T., Sakamoto, T., and Koumura, T. (2017). Lipid peroxidation-dependent cell death regulated by GPx4 and ferroptosis. Curr. Top. Microbiol. Immunol. 403, 143-170. doi: 10.1007/82_2016_508

Jiang, X., Stockwell, B. R., and Conrad, M. (2021). Ferroptosis: mechanisms, biology and role in disease. Nat. Rev. Mol. Cell Biol. 22, 266-282. doi: 10.1038/ s41580-020-00324-8

Kagan, V. E., Mao, G., Qu, F., Angeli, J. P., Doll, S., Croix, C. S., et al. (2017). Oxidized arachidonic and adrenic PEs navigate cells to ferroptosis. Nat. Chem. Biol. 13, 81-90. doi: 10.1038/nchembio. 2238
Kang, J. H., Kim, M. H., Lee, H. J., Huh, J. W., Lee, H. S., and Lee, D. S. (2020). Peroxiredoxin 4 attenuates glutamate-induced neuronal cell death through inhibition of endoplasmic reticulum stress. Free Radic. Res. 54, 207-220. doi: 10.1080/10715762.2020.1745201

Karuppagounder, S. S., Alin, L., Chen, Y., Brand, D., Bourassa, M. W., Dietrich, K., et al. (2018). N-acetylcysteine targets 5 lipoxygenase-derived, toxic lipids and can synergize with prostaglandin $\mathrm{E}(2)$ to inhibit ferroptosis and improve outcomes following hemorrhagic stroke in mice. Ann. Neurol. 84, 854-872. doi: 10.1002/ana.25356

Ke, Y., and Qian, Z. M. (2007). Brain iron metabolism: neurobiology and neurochemistry. Prog. Neurobiol. 83, 149-173. doi: 10.1016/j.pneurobio.2007. 07.009

Kenny, E. M., Fidan, E., Yang, Q., Anthonymuthu, T. S., New, L. A., Meyer, E. A., et al. (2019). Ferroptosis contributes to neuronal death and functional outcome after traumatic brain injury. Crit. Care Med. 47, 410-418. doi: 10.1097/ccm. 0000000000003555

Latunde-Dada, G. O. (2017). Ferroptosis: role of lipid peroxidation, iron and ferritinophagy. Biochim. Biophys. Acta Gen. Subj. 1861, 1893-1900. doi: 10. 1016/j.bbagen.2017.05.019

Le Corre, M., Noristani, H. N., Mestre-Frances, N., Saint-Martin, G. P., Coillot, C., Goze-Bac, C., et al. (2018). A novel translational model of spinal cord injury in nonhuman primate. Neurotherapeutics 15, 751-769. doi: 10.1007/s13311-0170589-9

Li, N., Jiang, W., Wang, W., Xiong, R., Wu, X., and Geng, Q. (2021). Ferroptosis and its emerging roles in cardiovascular diseases. Pharmacol. Res. 166:105466. doi: 10.1016/j.phrs.2021.105466

Liang, C., Zhang, X., Yang, M., and Dong, X. (2019). Recent progress in ferroptosis inducers for cancer therapy. Adv. Mater. 31:e1904197. doi: 10.1002/adma. 201904197

Ma, S., Dielschneider, R. F., Henson, E. S., Xiao, W., Choquette, T. R., Blankstein, A. R., et al. (2017). Ferroptosis and autophagy induced cell death occur independently after siramesine and lapatinib treatment in breast cancer cells. PLoS One 12:e0182921. doi: 10.1371/journal.pone.0182921

Maas, A. I. R., Menon, D. K., Adelson, P. D., Andelic, N., Bell, M. J., Belli, A., et al. (2017). Traumatic brain injury: integrated approaches to improve prevention, clinical care, and research. Lancet Neurol. 16, 987-1048. doi: 10.1016/s14744422(17)30371-x

Magtanong, L., and Dixon, S. J. (2018). Ferroptosis and brain injury. Dev. Neurosci. 40, 382-395. doi: 10.1159/000496922

Müller, T., Dewitz, C., Schmitz, J., Schröder, A. S., Bräsen, J. H., Stockwell, B. R., et al. (2017). Necroptosis and ferroptosis are alternative cell death pathways that operate in acute kidney failure. Cell. Mol. Life Sci. 74, 3631-3645. doi: 10.1007/s00018-017-2547-4

Neitemeier, S., Jelinek, A., Laino, V., Hoffmann, L., Eisenbach, I., Eying, R., et al. (2017). BID links ferroptosis to mitochondrial cell death pathways. Redox Biol. 12, 558-570. doi: 10.1016/j.redox.2017.03.007

Ng, S. Y., and Lee, A. Y. W. (2019). Traumatic brain injuries: Pathophysiology and potential therapeutic targets. Front. Cell. Neurosci. 13:528. doi: 10.3389/fncel. 2019.00528

Patil, N., Walsh, P., Carrabre, K., Holmberg, E. G., Lavoie, N., Dutton, J. R., et al. (2021). Regionally specific human pre-oligodendrocyte progenitor cells produce both oligodendrocytes and neurons after transplantation in a chronically injured spinal cord rat model after glial scar ablation. J. Neurotrauma 38, 777-788. doi: 10.1089/neu.2020.7009

Shen, L., Lin, D., Li, X., Wu, H., Lenahan, C., Pan, Y., et al. (2020). Ferroptosis in acute central nervous system injuries: the future direction? Front. Cell. Dev. Biol. 8:594. doi: 10.3389/fcell.2020.00594

Shi, Z., Yuan, S., Shi, L., Li, J., Ning, G., Kong, X., et al. (2021). Programmed cell death in spinal cord injury pathogenesis and therapy. Cell Prolif. 54:e12992. doi: $10.1111 /$ cpr.12992

Shichiri, M. (2014). The role of lipid peroxidation in neurological disorders. J. Clin. Biochem. Nutr. 54, 151-160. doi: 10.3164/jcbn.14-10

Snodgrass, R. G., and Brüne, B. (2019). Regulation and functions of 15lipoxygenases in human macrophages. Front. Pharmacol. 10:719. doi: 10.3389/ fphar.2019.00719

Spasić, S., Nikolić-Kokić, A., Miletić, S., Orešćanin-Dušić, Z., Spasić, M. B., Blagojević, D., et al. (2020). Edaravone may prevent ferroptosis in ALS. Curr. Drug Targets 21, 776-780. doi: 10.2174/1389450121666200220123305 
Stockwell, B. R., Friedmann Angeli, J. P., Bayir, H., Bush, A. I., Conrad, M., Dixon, S. J., et al. (2017). Ferroptosis: a regulated cell death nexus linking metabolism. Redox Biol. Dis. Cell 171, 273-285. doi: 10.1016/j.cell.2017.09.021

Tang, D., Chen, X., Kang, R., and Kroemer, G. (2021). Ferroptosis: molecular mechanisms and health implications. Cell Res. 31, 107-125. doi: 10.1038/ s41422-020-00441-1

Thayyullathil, F., Cheratta, A. R., Alakkal, A., Subburayan, K., Pallichankandy, S., Hannun, Y. A., et al. (2021). Acid sphingomyelinase-dependent autophagic degradation of GPX4 is critical for the execution of ferroptosis. Cell Death Dis. 12:26. doi: 10.1038/s41419-020-03297-w

Thorburne, S. K., and Juurlink, B. H. (1996). Low glutathione and high iron govern the susceptibility of oligodendroglial precursors to oxidative stress. J. Neurochem. 67, 1014-1022. doi: 10.1046/j.1471-4159.1996.67031014.x

Tobaben, S., Grohm, J., Seiler, A., Conrad, M., Plesnila, N., and Culmsee, C. (2011). Bid-mediated mitochondrial damage is a key mechanism in glutamateinduced oxidative stress and AIF-dependent cell death in immortalized HT22 hippocampal neurons. Cell Death Differ. 18, 282-292. doi: 10.1038/cdd. 2010.92

Wan, J., Ren, H., and Wang, J. (2019). Iron toxicity, lipid peroxidation and ferroptosis after intracerebral haemorrhage. Stroke Vasc. Neurol. 4, 93-95. doi: 10.1136/svn-2018-000205

Wu, Y., Song, J., Wang, Y., Wang, X., Culmsee, C., and Zhu, C. (2019). The potential role of ferroptosis in neonatal brain injury. Front. Neurosci. 13:115. doi: 10.3389/fnins.2019.00115

Xiao, X., Jiang, Y., Liang, W., Wang, Y., Cao, S., Yan, H., et al. (2019). miR-212-5p attenuates ferroptotic neuronal death after traumatic brain injury by targeting Ptgs2. Mol. Brain 12:78. doi: 10.1186/s13041-019-0501-0

Xie, B. S., Wang, Y. Q., Lin, Y., Mao, Q., Feng, J. F., Gao, G. Y., et al. (2019). Inhibition of ferroptosis attenuates tissue damage and improves long-term outcomes after traumatic brain injury in mice. CNS Neurosci. Ther. 25, 465-475. doi: $10.1111 / \mathrm{cns} .13069$

Xie, Y., Hou, W., Song, X., Yu, Y., Huang, J., Sun, X., et al. (2016). Ferroptosis: process and function. Cell Death Differ. 23, 369-379. doi: 10.1038/cdd. 2015.158
Yang, W. S., SriRamaratnam, R., Welsch, M. E., Shimada, K., Skouta, R., Viswanathan, V. S., et al. (2014). Regulation of ferroptotic cancer cell death by GPX4. Cell 156, 317-331. doi: 10.1016/j.cell.2013.12.010

Yao, X., Zhang, Y., Hao, J., Duan, H. Q., Zhao, C. X., Sun, C., et al. (2019). Deferoxamine promotes recovery of traumatic spinal cord injury by inhibiting ferroptosis. Neural Regen. Res. 14, 532-541. doi: 10.4103/1673-5374.245480

Yuan, H., Li, X., Zhang, X., Kang, R., and Tang, D. (2016). Identification of ACSL4 as a biomarker and contributor of ferroptosis. Biochem. Biophys. Res. Commun. 478, 1338-1343. doi: 10.1016/j.bbrc.2016.08.124

Zhang, Y., Sun, C., Zhao, C., Hao, J., Zhang, Y., Fan, B., et al. (2019). Ferroptosis inhibitor SRS 16-86 attenuates ferroptosis and promotes functional recovery in contusion spinal cord injury. Brain Res. 1706, 48-57. doi: 10.1016/j.brainres. 2018.10.023

Zhou, H., Yin, C., Zhang, Z., Tang, H., Shen, W., Zha, X., et al. (2020). Proanthocyanidin promotes functional recovery of spinal cord injury via inhibiting ferroptosis. J. Chem. Neuroanat. 107:101807. doi: 10.1016/j. jchemneu.2020.101807

Conflict of Interest: The authors declare that the research was conducted in the absence of any commercial or financial relationships that could be construed as a potential conflict of interest.

Publisher's Note: All claims expressed in this article are solely those of the authors and do not necessarily represent those of their affiliated organizations, or those of the publisher, the editors and the reviewers. Any product that may be evaluated in this article, or claim that may be made by its manufacturer, is not guaranteed or endorsed by the publisher.

Copyright (C) $2021 \mathrm{Hu}, \mathrm{Xu}, \mathrm{Xu}$, Jin, Zhang, Su, Li, Zhou and Ni. This is an open-access article distributed under the terms of the Creative Commons Attribution License (CC BY). The use, distribution or reproduction in other forums is permitted, provided the original author(s) and the copyright owner(s) are credited and that the original publication in this journal is cited, in accordance with accepted academic practice. No use, distribution or reproduction is permitted which does not comply with these terms. 